Article

\title{
Two-Variable Type 2 Poly-Fubini Polynomials
}

\author{
Ghulam Muhiuddin ${ }^{1}$, Waseem Ahmad Khan ${ }^{2}$ (D) and Ugur Duran ${ }^{3, *(D)}$ \\ 1 Department of Mathematics, University of Tabuk, Tabuk 71491, Saudi Arabia; chistygm@gmail.com or \\ gmuhiuddin@ut.edu.sa \\ 2 Department of Mathematics and Natural Sciences, Prince Mohammad Bin Fahd University, P.O. Box 1664, \\ Al Khobar 31952, Saudi Arabia; wkhan1@pmu.edu.sa \\ 3 Department of the Basic Concepts of Engineering, Faculty of Engineering and Natural Sciences, Iskenderun \\ Technical University, Hatay TR-31200, Turkey \\ * Correspondence: mtdrnugur@gmail.com or ugur.duran@iste.edu.tr
}

Citation: Muhiuddin, G.; Khan, W.A.; Duran, U. Two-Variable Type 2

Poly-Fubini Polynomials. Mathematics 2021, 9, 281. https://doi.org/ $10.3390 /$ math 9030281

Academic Editor: Francesco Aldo Costabile

Received: 30 December 2020

Accepted: 26 January 2021

Published: 31 January 2021

Publisher's Note: MDPI stays neutral with regard to jurisdictional clai$\mathrm{ms}$ in published maps and institutional affiliations.

Copyright: (C) 2021 by the authors. Licensee MDPI, Basel, Switzerland. This article is an open access article distributed under the terms and conditions of the Creative Commons Attribution (CC BY) license (https:// creativecommons.org/licenses/by/ $4.0 /)$.

\begin{abstract}
In the present work, a new extension of the two-variable Fubini polynomials is introduced by means of the polyexponential function, which is called the two-variable type 2 poly-Fubini polynomials. Then, some useful relations including the Stirling numbers of the second and the first kinds, the usual Fubini polynomials, and the higher-order Bernoulli polynomials are derived. Also, some summation formulas and an integral representation for type 2 poly-Fubini polynomials are investigated. Moreover, two-variable unipoly-Fubini polynomials are introduced utilizing the unipoly function, and diverse properties involving integral and derivative properties are attained. Furthermore, some relationships covering the two-variable unipoly-Fubini polynomials, the Stirling numbers of the second and the first kinds, and the Daehee polynomials are acquired.
\end{abstract}

Keywords: polyexponential function; Fubini polynomials; poly-Fubini polynomials; unipoly function; Stirling numbers

\section{Introduction}

Throughout the paper, we use $\mathbb{N}:=\{1,2,3, \cdots\}$ and $\mathbb{N}_{0}=\mathbb{N} \cup\{0\}$. Let $\mathbb{C}$ denote the set of complex numbers, $\mathbb{R}$ denote the set of real numbers, and $\mathbb{Z}$ denote the set of integers.

The usual Euler $E_{n}(x)$ and Bernoulli polynomials $B_{n}(x)$ are defined via the following exponential generating functions (cf. [1-6]):

$$
\frac{2}{e^{z}+1} e^{x z}=\sum_{n=0}^{\infty} E_{n}(x) \frac{z^{n}}{n !}(|z|<\pi) \text { and } \frac{z}{e^{z}-1} e^{x z}=\sum_{n=0}^{\infty} B_{n}(x) \frac{z^{n}}{n !}(|z|<2 \pi) .
$$

The two-variable Fubini polynomials are defined as follows (cf. [1,2,4,7-10]):

$$
\frac{e^{x z}}{1-y\left(e^{z}-1\right)}=\sum_{n=0}^{\infty} F_{n}(x, y) \frac{z^{n}}{n !}
$$

Substituting $x=0$ in (2), we have $F_{n}(0, y):=F_{n}(y)$ called the usual Fubini polynomials given by

$$
\frac{1}{1-y\left(e^{z}-1\right)}=\sum_{n=0}^{\infty} F_{n}(y) \frac{z^{n}}{n !}
$$

It is easy to see from (1) and (2) that

$$
F_{n}\left(x,-\frac{1}{2}\right)=E_{n}(x)
$$

Upon letting $y=1$ in (3), we get the Fubini numbers as follows 


$$
\frac{1}{2-e^{z}}=\sum_{n=0}^{\infty} F_{n} \frac{z^{n}}{n !}
$$

For more detailed information of the Fubini polynomials with applications, see $[1,2,4,7-10]$.

The Bernoulli polynomials of the second kind are defined as follows (cf. $[5,11,12])$ :

$$
\frac{z}{\log (1+z)}(1+z)^{x}=\sum_{n=0}^{\infty} b_{n}(x) \frac{z^{n}}{n !}
$$

The Bernoulli polynomials of order $\alpha \in \mathbb{N}$ are defined by (cf. [5,6,11-13])

$$
\left(\frac{z}{e^{z}-1}\right)^{\alpha} e^{x z}=\sum_{n=0}^{\infty} B_{n}^{(\alpha)}(x) \frac{z^{n}}{n !}
$$

By (5) and (6),

$$
B_{n}^{(n)}(x+1):=b_{n}(x) .
$$

The polyexponential function $\mathrm{Ei}_{k}(x)$ is introduced by Kim-Kim [12] as follows

$$
\operatorname{Ei}_{k}(x)=\sum_{n=1}^{\infty} \frac{x^{n}}{(n-1) ! n^{k^{\prime}}}, \quad(k \in \mathbb{Z})
$$

as inverse the polylogarithm function $L i_{k}(z)$ (cf. [6,13-15]) given by

$$
L i_{k}(z)=\sum_{n=1}^{\infty} \frac{z^{n}}{n^{k}},|z|<1
$$

Using the polyexponential function $\mathrm{Ei}_{k}(x), \mathrm{Kim}$-Kim [12] considered type 2 poly-Bernoulli polynomials, given by

$$
\frac{\mathrm{Ei}_{k}(\log (1+z))}{e^{z}-1} e^{x z}=\sum_{n=0}^{\infty} \beta_{n}^{(k)}(x) \frac{z^{n}}{n !}, \quad(k \in \mathbb{Z})
$$

and attained several properties and formulas for these polynomials. Upon setting $x=0$ in $(10), \beta_{n}^{(k)}(0):=\beta_{n}^{(k)}$ are called type 2 poly-Bernoulli numbers.

We also notice that $\operatorname{Ei}_{1}(z)=e^{z}-1$. Hence, when $k=1$, the type 2 poly-Bernoulli $\beta_{n}^{(k)}(x)$ polynomials reduce to the Bernoulli polynomials $B_{n}(x)$ in (1).

Some mathematicians have considered and examined several extensions of special polynomials via polyexponential function, $\mathrm{cf}$. $[5,11,13,16,17]$ and see also the references cited therein. For example, Duran et al. [11] defined type 2 poly-Frobenius-Genocchi polynomials by the following Maclaurin series expansion (in a suitable neighborhood of $z=0)$ :

$$
\frac{\operatorname{Ei}_{k}(\log (1+(1-u) z))}{e^{z}-u} e^{x z}=\sum_{n=0}^{\infty} G_{n}^{(F, k)}(x ; u) \frac{z^{n}}{n !} \quad(k \in \mathbb{Z})
$$

and Lee et al. [17] introduced type 2 poly-Euler polynomials given by

$$
\frac{\mathrm{Ei}_{k}(\log (1+2 z))}{z\left(e^{z}+1\right)} e^{x z}=\sum_{n=0}^{\infty} \mathcal{E}_{n}^{(k)}(x) \frac{z^{n}}{n !}
$$

Kim-Kim [12] also introduced unipoly function $u_{k}(x \mid p)$ attached to $p$ being any arithmetic map which is a complex or real-valued function defined on $\mathbb{N}$ as follows:

$$
u_{k}(x \mid p)=\sum_{n=1}^{\infty} \frac{p(n)}{n^{k}} x^{n}, \quad(k \in \mathbb{Z})
$$


It is readily seen that

$$
u_{k}(x \mid 1)=\sum_{n=1}^{\infty} \frac{x^{n}}{n^{k}}=L i_{k}(x)
$$

is the ordinary polylogarithm function in (9). By utilizing the unipoly function $u_{k}(x \mid p)$, Kim-Kim [12] defined unipoly-Bernoulli polynomials as follows:

$$
\sum_{n=0}^{\infty} B_{n, p}^{(k)}(x) \frac{z^{n}}{n !}=\frac{u_{k}\left(1-e^{-z} \mid p\right)}{1-e^{-z}} e^{x z} .
$$

They derived diverse formulas and relationships for these polynomials, see [12].

The Stirling numbers of the first kind $S_{1}(n, k)$ and the second kind $S_{2}(n, k)$ are given below:

$$
\frac{(\log (1+z))^{k}}{k !}=\sum_{n=0}^{\infty} S_{1}(n, k) \frac{z^{n}}{n !} \text { and } \frac{\left(e^{z}-1\right)^{k}}{k !}=\sum_{n=0}^{\infty} S_{2}(n, k) \frac{z^{n}}{n !} .
$$

From (13), for $n \geq 0$, we obtain

$$
(x)_{n}=\sum_{k=0}^{n} S_{1}(n, k) x^{k} \text { and } x^{n}=\sum_{k=0}^{n} S_{1}(n, k)(x)_{k^{\prime}}
$$

where $(x)_{0}=1$ and $(x)_{n}=x(x-1)(x-2) \cdots(x-n+1)$, cf. [1-4,6-9,12-15].

From (3) and (13), we get

$$
F_{n}(y)=\sum_{k=0}^{n} S_{2}(n, k) k ! y^{k}
$$

In the following sections, we introduce a new extension of the two-variable Fubini polynomials by means of the polyexponential function, which we call two-variable type 2 poly-Fubini polynomials. Then, we derive some useful relations including the Stirling numbers of the first and the second kinds, the usual Fubini polynomials, and the Bernoulli polynomials of higher-order. Also, we investigate some summation formulas and an integral representation for type 2 poly-Fubini polynomials. Moreover, we introduce twovariable unipoly-Fubini polynomials via unipoly function and acquire diverse properties including derivative and integral properties. Furthermore, we provide some relationships covering the Stirling numbers of the first and the second kinds, the two-variable unipolyFubini polynomials, and the Daehee polynomials.

\section{Two-Variable Type 2 Poly-Fubini Polynomials and Numbers}

Inspired and motivated by the definition of type 2 poly-Bernoulli polynomials in (10) given by Kim-Kim [12], here, we introduce two-variable type 2 poly-Fubini polynomials by Definition 1 as follows.

Definition 1. For $k \in \mathbb{Z}$, we define two-variable type 2 poly-Fubini polynomials via the following exponential generating function (in a suitable neighborhood of $z=0$ ) as given below:

$$
\frac{\operatorname{Ei}_{k}(\log (1+z))}{z\left(1-y\left(e^{z}-1\right)\right)} e^{x z}=\sum_{n=0}^{\infty} F_{n}^{(k)}(x ; y) \frac{z^{n}}{n !} .
$$

Upon setting $x=0$ in (16), we have $F_{n}^{(k)}(0 ; y):=F_{n}^{(k)}(y)$ which we call type 2 poly-Fubini polynomials possessing the following generating function: 


$$
\frac{\operatorname{Ei}_{k}(\log (1+z))}{z\left(1-y\left(e^{z}-1\right)\right)}=\sum_{n=0}^{\infty} F_{n}^{(k)}(y) \frac{z^{n}}{n !} .
$$

We note that, for $k=1$, the two-variable type 2 poly-Fubini polynomials reduce to the usual two-variable Fubini polynomials in (2) because of $\operatorname{Ei}_{1}(z)=e^{z}-1$.

Now, we develop some relationships and formulas for two-variable type 2 poly-Fubini polynomials as follows.

Theorem 1. The following relationship

$$
F_{n}^{(k)}(x ; y)=\sum_{l=0}^{n}\left(\begin{array}{l}
n \\
l
\end{array}\right) F_{n-l}^{(k)}(y)(u) x^{l}
$$

holds for $k \in \mathbb{Z}$ and $n \geq 0$.

Proof. By (16) and (17), we consider that

$$
\begin{aligned}
\sum_{n=0}^{\infty} F_{n}^{(k)}(x ; y) \frac{z^{n}}{n !} & =\frac{\operatorname{Ei}_{k}(\log (1+z))}{z\left(1-y\left(e^{z}-1\right)\right)} e^{x z} \\
& =\sum_{n=0}^{\infty} \frac{x^{n} z^{n}}{n !} \sum_{n=0}^{\infty} F_{n}^{(k)}(y) \frac{z^{n}}{n !} \\
& =\sum_{n=0}^{\infty}\left(\sum_{l=0}^{n}\left(\begin{array}{l}
n \\
l
\end{array}\right) F_{n-l}^{(k)}(y)(u) x^{l}\right) \frac{z^{n}}{n !},
\end{aligned}
$$

which gives the asserted result (18).

A relationship involving Stirling numbers of the first kind, the two-variable Fubini polynomials, and two-variable type 2 poly-Fubini polynomials is stated by the following theorem.

Theorem 2. For $k \in \mathbb{Z}$ and $n \geq 0$, we have

$$
F_{n}^{(k)}(x ; y)=\sum_{l=0}^{n} \sum_{m=0}^{l}\left(\begin{array}{l}
n \\
l
\end{array}\right) \frac{S_{1}(l+1, m+1)}{(m+1)^{k-1}} \frac{F_{n-l}(x ; y)}{l+1} .
$$

Proof. From (13) and (17), we observe that

$$
\begin{aligned}
\sum_{n=0}^{\infty} F_{n}^{(k)}(x ; y) \frac{z^{n}}{n !} & =\frac{e^{x z}}{z\left(1-y\left(e^{z}-1\right)\right)} \sum_{m=1}^{\infty} \frac{(\log (1+z))^{m}}{(m-1) ! m^{k}} \\
& =\frac{e^{x z}}{z\left(1-y\left(e^{z}-1\right)\right)} \sum_{m=0}^{\infty} \frac{(\log (1+z))^{m+1}}{(m+1)^{k}} \frac{1}{m !} \\
& =\sum_{n=0}^{\infty} F_{n}(x ; y) \frac{z^{n}}{n !} \sum_{n=0}^{\infty} \sum_{n=m}^{\infty} \frac{1}{n+1} \frac{S_{1}(n+1, m+1)}{(m+1)^{k-1}} \frac{z^{n}}{n !} \\
& =\sum_{n=0}^{\infty}\left(\sum_{l=0}^{n} \sum_{m=0}^{l}\left(\begin{array}{l}
n \\
l
\end{array}\right) \frac{S_{1}(l+1, m+1)}{(m+1)^{k-1}} \frac{F_{n-l}(x ; y)}{l+1}\right) \frac{z^{n}}{n !},
\end{aligned}
$$

which means the desired result (19).

Some special cases of Theorem 2 are examined below.

Corollary 1. For $k \in \mathbb{Z}$ and $n \geq 0$, we get 


$$
F_{n}^{(k)}(y)=\sum_{l=0}^{n} \sum_{m=0}^{l}\left(\begin{array}{l}
n \\
l
\end{array}\right) \frac{S_{1}(l+1, m+1)}{l+1} \frac{F_{n-l}(y)}{(m+1)^{k-1}} .
$$

Corollary 2. For $k=1$ and $n \geq 0$, we acquire

$$
F_{n}(x ; y)=\sum_{l=0}^{n} \sum_{m=0}^{l}\left(\begin{array}{l}
n \\
l
\end{array}\right) \frac{F_{n-l}(x ; y)}{l+1} S_{1}(l+1, m+1) .
$$

The following differentiation property holds (cf. [12])

$$
\frac{d}{d x} \operatorname{Ei}_{k}(\log (1+x))=\frac{1}{(1+x) \log (1+x)} \operatorname{Ei}_{k-1}(\log (1+x) .
$$

and also, the following integral representations are valid for $k>1$ :

$$
\begin{aligned}
\operatorname{Ei}_{k}(\log (1+x))= & \int_{0}^{x} \frac{1}{(1+z) \log (1+z)} \\
& \times \underbrace{\int_{0}^{z} \frac{1}{(1+z) \log (1+z)} \cdots \int_{0}^{z} \frac{z}{(1+z) \log (1+z)}}_{(k-2) \text { times }} d z d z \ldots d z \\
= & x \sum_{m=0}^{\infty} \sum_{m_{1}+\cdots+m_{k-1}=m}\left(m_{1}, \cdots, m_{k-1}\right) \\
& \times \frac{B_{m_{1}}^{\left(m_{1}\right)}}{m_{1}+1} \frac{B_{m_{2}}^{\left(m_{2}\right)}}{m_{1}+m_{2}+1} \cdots \frac{B_{m_{k-1}}^{\left(m_{k-1}\right)}}{m_{1}+\cdots+m_{k-1}+1} \frac{x^{m}}{m !} .
\end{aligned}
$$

Theorem 3. The following relationship

$$
\begin{aligned}
F_{n}^{(k)}(y)= & \sum_{m=0}^{n}\left(\begin{array}{c}
n \\
m
\end{array}\right) \sum_{m_{1}+\cdots+m_{k-1}=m}\left(\begin{array}{c}
m \\
m_{1}, \cdots, m_{k-1}
\end{array}\right) \\
& \times \frac{B_{m_{1}}^{\left(m_{1}\right)}}{m_{1}+1} \frac{B_{m_{2}}^{\left(m_{2}\right)}}{m_{1}+m_{2}+1} \cdots \frac{B_{m_{k-1}}^{\left(m_{k-1}\right)}}{m_{1}+\cdots+m_{k-1}+1} F_{n-m}(y)
\end{aligned}
$$

holds for $n \in \mathbb{N}_{0}$ and $k>1$.

Proof. From (17) and (21), for $k>1$, we can write

$$
\begin{gathered}
\sum_{n=0}^{\infty} F_{n}^{(k)}(y) \frac{z^{n}}{n !}=\frac{\operatorname{Ei}_{k}(\log (1+z))}{z\left(1-y\left(e^{z}-1\right)\right)} \\
=\frac{z}{z\left(1-y\left(e^{z}-1\right)\right)} \sum_{m=0}^{\infty} \sum_{m_{1}+\cdots+m_{k-1}=m}\left(\begin{array}{c}
m \\
m_{1}, \cdots, m_{k-1}
\end{array}\right) \\
\times \frac{B_{m_{1}}^{\left(m_{1}\right)}}{m_{1}+1} \frac{B_{m_{2}}^{\left(m_{2}\right)}}{m_{1}+m_{2}+1} \cdots \frac{B_{m_{k-1}}^{\left(m_{k-1}\right)}}{m_{1}+\cdots+m_{k-1}+1} \frac{x^{m}}{m !}
\end{gathered}
$$

Theorem 4. The following relationship

$$
F_{n}^{(k)}(y)=\frac{1}{1+y}\left(y \sum_{m=0}^{n}\left(\begin{array}{c}
n \\
m
\end{array}\right) F_{n-m}^{(k)}(y)+\sum_{m=0}^{n} \frac{1}{(m+1)^{k-1}} \frac{S_{1}(n+1, m+1)}{n+1}\right)
$$

holds for $n \geq 0$. 
Proof. From (17), we attain

$$
\begin{gathered}
\frac{\operatorname{Ei}_{k}(\log (1+z))}{z}=\sum_{n=0}^{\infty} F_{n}^{(k)}(y) \frac{z^{n}}{n !}\left(1-y\left(e^{z}-1\right)\right) \\
=\sum_{n=0}^{\infty} F_{n}^{(k)}(y) \frac{z^{n}}{n !}-y \sum_{n=0}^{\infty} \sum_{m=0}^{n}\left(\begin{array}{c}
n \\
m
\end{array}\right) F_{n-m}^{(k)}(y) \frac{z^{n}}{n !}+y \sum_{n=0}^{\infty} F_{n}^{(k)}(y) \frac{z^{n}}{n !} \\
=\sum_{n=0}^{\infty}\left((1+y) F_{n}^{(k)}(y)-y \sum_{m=0}^{n}\left(\begin{array}{c}
n \\
m
\end{array}\right) F_{n-m}^{(k)}(y)\right) \frac{z^{n}}{n !}
\end{gathered}
$$

and, also, we have

$$
\begin{gathered}
\operatorname{Ei}_{k}(\log (1+z))=\frac{1}{z} \sum_{m=1}^{\infty} \frac{(\log (1+z))^{m}}{m^{k}} \frac{1}{(m-1) !} \\
=\frac{1}{z} \sum_{m=0}^{\infty} \frac{(\log (1+z))^{m+1}}{(m+1)^{k}} \frac{1}{m !}=\frac{1}{z} \sum_{m=0}^{\infty} \frac{(\log (1+z))^{m+1}}{(m+1)^{k-1}} \frac{1}{(m+1) !} \\
=\frac{1}{z} \sum_{m=0}^{\infty} \sum_{n=m+1}^{\infty} \frac{S_{1}(n, m+1)}{(m+1)^{k-1}} \frac{z^{n}}{n !},
\end{gathered}
$$

which implies the asserted result (22).

For $s \in \mathbb{C}$ and $k \in \mathbb{Z}$ with $k \geq 1$, let

$$
\eta_{k}(s):=\frac{1}{\Gamma(s)} \int_{0}^{\infty} \frac{z^{s-1}}{z\left(1-y\left(e^{z}-1\right)\right)} \operatorname{Ei}_{k}(\log (1+z)) d z,
$$

where $\Gamma(s)$ is the classical gamma function given below:

$$
\Gamma(s)=\int_{0}^{\infty} z^{s-1} e^{z} d z \quad(\Re(s)>0) .
$$

From (23), we see that $\eta_{k}(s)$ is a holomorphic map for $\Re(s)>0$, since $\operatorname{Ei}_{k}(\log (1+z)) \leq$ $\mathrm{Ei}_{1}(\log (1+z))$ with $z \geq 0$. Thus, we have

$$
\eta_{k}(s)=\frac{1}{\Gamma(s)} \int_{0}^{1} \frac{z^{s-2}}{1-y\left(e^{z}-1\right)} \operatorname{Ei}_{k}(\log (1+z)) d z+\frac{1}{\Gamma(s)} \int_{1}^{\infty} \frac{z^{s-2}}{1-y\left(e^{z}-1\right)} \operatorname{Ei}_{k}(\log (1+z)) d z
$$

We see that the second integral in (24) converges absolutely for any $s \in \mathbb{C}$ and hence, the second term on the right hand side vanishes at non-positive integers. Therefore, we obtain

$$
\lim _{s \rightarrow-m}\left|\frac{1}{\Gamma(s)} \int_{1}^{\infty} \frac{z^{s-2}}{1-y\left(e^{z}-1\right)} \operatorname{Ei}_{k}(\log (1+z)) d z\right| \leq \frac{1}{\Gamma(-m)} M=0,
$$

since

$$
\Gamma(s) \Gamma(1-s)=\frac{\pi}{\sin (\pi s)} .
$$

Also, for $\Re(s)>0$, the first integral in (24) can be written as

$$
\begin{gathered}
\frac{1}{\Gamma(s)} \int_{0}^{1} \frac{z^{s-1}}{z\left(1-y\left(e^{z}-1\right)\right)} \operatorname{Ei}_{k}(\log (1+z)) d z=\frac{1}{\Gamma(s)} \sum_{n=0}^{\infty} \frac{F_{n}^{(k)}(y)}{n !} \int_{0}^{1} z^{n+s-1} d z \\
=\frac{1}{\Gamma(s)} \sum_{n=0}^{\infty} \frac{F_{n}^{(k)}(y)}{n !} \frac{1}{n+s},
\end{gathered}
$$

which defines an entire function of $s$. Therefore, we derive that $\eta_{k}(s)$ can be continued to an entire map of $s$. 
Theorem 5. For $k \in \mathbb{N}$, the map $\eta_{k}(s)$ has an analytic continuation to a map of $s \in \mathbb{C}$, and the special values at non-positive integers are as follows

$$
\eta_{k}(-m)=(-1)^{m} F_{m}^{(k)}(y),\left(m \in \mathbb{N}_{0}\right) .
$$

Proof. By means of (24)-(26), we acquire

$$
\begin{aligned}
\eta_{k}(-m) & =\lim _{s \rightarrow-m} \frac{1}{\Gamma(s)} \int_{0}^{1} \frac{z^{s-1}}{z\left(1-y\left(e^{z}-1\right)\right)} \operatorname{Ei}_{k}(\log (1+z)) d z \\
& =\cdots+\cdots+0+\lim _{s \rightarrow-m} \frac{1}{\Gamma(s)} \frac{F_{m}^{(k)}}{m !(m+s)}+0+0+\cdots \\
& =\lim _{s \rightarrow-m} \frac{1}{m+s} \frac{\Gamma(1-s) \sin (\pi s)}{\pi} \frac{F_{m}^{(k)}(y)}{m !} \\
& =\frac{\Gamma(1+m)}{m !} \cos (\pi m) F_{m}^{(k)}(y)=(-1)^{m} F_{m}^{(k)}(y)
\end{aligned}
$$

which is the desired relation in (27).

Now, we state a summation formula for $F_{n}^{(k)}(x ; y)$ as given below.

Theorem 6. The following formula

$$
F_{n}^{(k)}\left(x_{1}+x_{2} ; y\right)=\sum_{m=0}^{n}\left(\begin{array}{c}
n \\
m
\end{array}\right) F_{n-m}^{(k)}\left(x_{1} ; y\right) x_{2}^{m}
$$

holds for $k \in \mathbb{Z}$ and $n \geq 0$.

Proof. By (17), we observe that

$$
\begin{aligned}
\sum_{n=0}^{\infty} F_{n}^{(k)}\left(x_{1}+x_{2} ; y\right) \frac{z^{n}}{n !} & =\left(\frac{\operatorname{Ei}_{k}(\log (1+z))}{z\left(1-y\left(e^{z}-1\right)\right)}\right) e^{\left(x_{1}+x_{2}\right) z} \\
& =\left(\sum_{n=0}^{\infty} F_{n}^{(k)}\left(x_{1} ; y\right) \frac{z^{n}}{n !}\right)\left(\sum_{m=0}^{\infty} x_{2}^{m} \frac{z^{m}}{m !}\right) \\
& =\sum_{n=0}^{\infty}\left(\sum_{m=0}^{n}\left(\begin{array}{c}
n \\
m
\end{array}\right) F_{n-m}^{(k)}\left(x_{1} ; y\right) x_{2}^{m}\right) \frac{z^{n}}{n !},
\end{aligned}
$$

which means the claimed result (28).

Theorem 7. The following formula

$$
y F_{n}^{(k)}(x+1 ; y)=(1+y) F_{n}^{(k)}(x ; y)-\sum_{l=0}^{n} \sum_{m=0}^{l}\left(\begin{array}{l}
n \\
l
\end{array}\right) \frac{1}{(m+1)^{k-1}} \frac{S_{1}(l+1, m+1)}{l+1} x^{n-l}
$$

is valid for $k \in \mathbb{Z}$ and $n \geq 0$.

Proof. By (14) and (17), we consider that 


$$
\begin{gathered}
\sum_{n=0}^{\infty}\left(F_{n}^{(k)}(x+1 ; y)-F_{n}^{(k)}(x ; y)\right) \frac{z^{n}}{n !}=e^{x z}\left(e^{z}-1\right) \frac{\operatorname{Ei}_{k}(\log (1+z))}{z\left(1-y\left(e^{z}-1\right)\right)} \\
=\left(\frac{e^{x z}}{1-y\left(e^{z}-1\right)}-e^{x z}\right) \frac{\operatorname{Ei}_{k}(\log (1+z))}{y z} \\
=\frac{1}{y}\left(\sum_{n=0}^{\infty} F_{n}^{(k)}(x ; y)-\sum_{l=0}^{n} \sum_{m=0}^{l}\left(\begin{array}{l}
n \\
l
\end{array}\right) \frac{x^{n-l}}{l+1} \frac{S_{1}(l+1, m+1)}{(m+1)^{k-1}}\right) \frac{z^{n}}{n !}
\end{gathered}
$$

which means the desired result (29).

Theorem 8. The following formula

$$
\sum_{m=0}^{n}\left(\begin{array}{l}
n \\
m
\end{array}\right) F_{n-m}^{(k)}\left(x_{1} ; y_{1}\right) F_{m}^{(k)}\left(x_{2} ; y_{2}\right)=\frac{y_{2} F_{n}^{(k)}\left(x_{1}+x_{2} ; y_{2}\right)-y_{1} F_{n}^{(k)}\left(x_{1}+x_{2} ; y_{1}\right)}{y_{2}-y_{1}}
$$

holds for $k \in \mathbb{Z}$ and $n \geq 0$.

Proof. By means of (17), we acquire

$$
\begin{gathered}
\mathrm{Y}=\sum_{n=0}^{\infty} F_{n}^{(k)}\left(x_{1} ; y_{1}\right) \frac{z^{n}}{n !} \sum_{n=0}^{\infty} F_{n}^{(k)}\left(x_{2} ; y_{2}\right) \frac{z^{n}}{n !}=\frac{\operatorname{Ei}_{k}(\log (1+z))}{z\left(1-y_{1}\left(e^{z}-1\right)\right)} e^{x_{1} z} \frac{\operatorname{Ei}_{k}(\log (1+z))}{z\left(1-y_{2}\left(e^{z}-1\right)\right)} e^{x_{2} z} \\
=\frac{\operatorname{Ei}_{k}(\log (1+z))}{z}\left(\frac{e^{x_{1} z}}{1-y_{1}\left(e^{z}-1\right)} \frac{e^{x_{2} z}}{1-y_{2}\left(e^{z}-1\right)}\right) \\
=\frac{\operatorname{Ei}_{k}(\log (1+z))}{z}\left(\frac{y_{2}}{y_{2}-y_{1}} \frac{e^{\left(x_{1}+x_{2}\right) z}}{1-y_{2}\left(e^{z}-1\right)}-\frac{y_{1}}{y_{2}-y_{1}} \frac{e^{\left(x_{1}+x_{2}\right) z}}{1-y_{1}\left(e^{z}-1\right)}\right) \\
=\sum_{n=0}^{\infty}\left(\frac{y_{2} F_{n}^{(k)}\left(x_{1}+x_{2} ; y_{2}\right)-y_{1} F_{n}^{(k)}\left(x_{1}+x_{2} ; y_{1}\right)}{y_{2}-y_{1}}\right) \frac{z^{n}}{n !}
\end{gathered}
$$

and

$$
\mathrm{Y}=\sum_{n=0}^{\infty}\left(\sum_{m=0}^{n}\left(\begin{array}{l}
n \\
m
\end{array}\right) F_{n-m}^{(k)}\left(x_{1} ; y_{1}\right) F_{m}^{(k)}\left(x_{2} ; y_{2}\right)\right) \frac{z^{n}}{n !},
$$

which means the claimed result (30).

Theorem 9. The following relationship

$$
F_{n}^{(k)}(y)=\sum_{l=0}^{n} \sum_{m=0}^{n-l} \sum_{r=0}^{l}\left(\begin{array}{l}
n \\
l
\end{array}\right) \frac{S_{1}(n-l+1, m+1) S_{2}(l, r)}{n-l+1} \frac{y^{r} r !}{(m+1)^{k-1}}
$$

holds for $k \in \mathbb{Z}$ and $n \geq 0$.

Proof. Using (18), we get

$$
\begin{aligned}
& \sum_{n=0}^{\infty} F_{n}^{(k)}(y) \frac{z^{n}}{n !}=\frac{\operatorname{Ei}_{k}(\log (1+z))}{z} \sum_{m=0}^{\infty} y^{m} \sum_{l=m}^{\infty} m ! S_{2}(l, m) \frac{z^{l}}{l !} \\
= & \left(\sum_{n=0}^{\infty} \sum_{m=0}^{n} \frac{S_{1}(n+1, m+1)}{(m+1)^{k-1}} \frac{z^{n}}{(n+1) !}\right)\left(\sum_{l=0}^{\infty} \sum_{r=0}^{l} y^{r} r ! S_{2}(l, r) \frac{z^{l}}{l !}\right) \\
= & \sum_{n=0}^{\infty}\left(\sum_{l=0}^{n} \sum_{m=0}^{n-l} \sum_{r=0}^{l}\left(\begin{array}{c}
n \\
l
\end{array}\right) S_{2}(l, r) \frac{S_{1}(n-l+1, m+1)}{n-l+1} \frac{y^{r} r !}{(m+1)^{k-1}}\right) \frac{z^{n}}{n !}
\end{aligned}
$$

which means the desired result (31). 
Theorem 10. The following correlation

$$
\sum_{m=0}^{n} F_{m}^{(k)}(y) S_{2}(n, m)=\sum_{q=0}^{n} \sum_{i=0}^{q} \sum_{p=0}^{i}\left(\begin{array}{l}
q \\
i
\end{array}\right)\left(\begin{array}{l}
n \\
q
\end{array}\right) F_{p}(y) S_{2}(i, p) B_{q-i} \frac{1}{(n-q+1)^{k}}
$$

hold for $k \in \mathbb{Z}$ and $n \geq 0$.

Proof. Using (18), replacing $z$ by $e^{z}-1$, we acquire that

$$
\begin{gathered}
\sum_{n=0}^{\infty} F_{n}^{(k)}(y) \frac{\left(e^{z}-1\right)^{n}}{n !}=\frac{1}{1-y\left(e^{z^{z}-1}-1\right)} \frac{z}{e^{z}-1} \frac{\mathrm{Ei}_{k}(z)}{z} \\
=\sum_{p=0}^{\infty} F_{p}(y) \sum_{p=i}^{\infty} S_{2}(i, p) \frac{z^{i}}{i !} \sum_{q=0}^{\infty} B_{q} \frac{z^{q}}{q !} \sum_{n=0}^{\infty} \frac{z^{n}}{n !(n+1)^{k}} \\
=\sum_{i=0}^{\infty}\left(\sum_{p=0}^{i} F_{p}(y) S_{2}(i, p)\right) \frac{z^{i}}{i !} \sum_{q=0}^{\infty} B_{q} \frac{z^{q}}{q !} \sum_{n=0}^{\infty} \frac{z^{n}}{n !(n+1)^{k}} \\
=\sum_{n=0}^{\infty}\left(\sum_{q=0}^{n} \sum_{i=0}^{q} \sum_{p=0}^{i}\left(\begin{array}{c}
n \\
q
\end{array}\right)\left(\begin{array}{l}
q \\
i
\end{array}\right) F_{p}(y) S_{2}(i, p) B_{q-i} \frac{1}{(n-q+1)^{k}}\right) \frac{z^{n}}{n !}
\end{gathered}
$$

and

$$
\sum_{m=0}^{\infty} F_{m}^{(k)}(y) \frac{\left(e^{z}-1\right)^{m}}{m !}=\sum_{m=0}^{\infty} F_{m}^{(k)}(y) \sum_{n=m}^{\infty} S_{2}(n, m) \frac{z^{n}}{n !}=\sum_{n=0}^{\infty}\left(\sum_{m=0}^{n} F_{m}^{(k)}(y) S_{2}(n, m)\right) \frac{z^{n}}{n !},
$$

which provides the asserted result (32).

\section{Two-Variable Unipoly-Fubini Polynomials}

Using the unipoly function $u_{k}(z \mid p)$ in (11), we introduce two-variable unipoly-Fubini polynomials attached to $p$ via the following generating function:

$$
\frac{u_{k}(\log (1+z) \mid p)}{z\left(1-y\left(e^{z}-1\right)\right)} e^{x z}=\sum_{n=0}^{\infty} F_{n, p}^{(k)}(x ; y) \frac{z^{n}}{n !} .
$$

Upon setting $x=0$ in (33), we have $F_{n, p}^{(k)}(0 ; y):=F_{n, p}^{(k)}(y)$ which we call unipoly-Fubini polynomials attached to $p$ as follows

$$
\frac{u_{k}(\log (1+z) \mid p)}{z\left(1-y\left(e^{z}-1\right)\right)}=\sum_{n=0}^{\infty} F_{n, p}^{(k)}(y) \frac{z^{n}}{n !} .
$$

We now investigate some properties of two-variable unipoly-Fubini polynomials attached to $p$ as follows.

Theorem 11. The following relationship

$$
F_{n, p}^{(k)}(x ; y)=\sum_{l=0}^{n}\left(\begin{array}{l}
n \\
l
\end{array}\right) F_{n-l, p}^{(k)}(y) x^{l}
$$

holds for $k \in \mathbb{Z}$ and $n \geq 0$.

Proof. By (33) and (34), we consider thatwhich gives the asserted result (35).

Theorem 12. The following derivative rule

$$
\frac{d}{d x} F_{n, p}^{(k)}(x ; y)=n F_{n-1, p}^{(k)}(x ; y)
$$


holds for $k \in \mathbb{Z}$ and $n \geq 1$.

Proof. From (33), we observe that

$$
\sum_{n=1}^{\infty} \frac{d}{d x} F_{n, p}^{(k)}(x ; y) \frac{z^{n}}{n !}=\frac{1}{z\left(1-y\left(e^{z}-1\right)\right)} u_{k}(\log (1+z) \mid p) \frac{d}{d x} e^{x z}=\sum_{n=0}^{\infty} F_{n, p}^{(k)}(x ; y) \frac{z^{n+1}}{n !},
$$

which means the desired result (36).

Theorem 13. The following integral representation

$$
\int_{\alpha}^{\beta} F_{n, p}^{(k)}(x ; y) d x=\frac{F_{n+1, p}^{(k)}(\beta ; y)-F_{n+1, p}^{(k)}(\alpha ; y)}{n+1}
$$

holds for $n \geq 0$ and $k \in \mathbb{Z}$.

Proof. By Theorem 12, we derive that

$$
\int_{\alpha}^{\beta} F_{n, p}^{(k)}(x ; y) d x=\frac{1}{n+1} \int_{\alpha}^{\beta} \frac{d}{d x} F_{n+1, p}^{(k)}(x ; y) d x=\frac{F_{n+1, p}^{(k)}(\alpha ; y)-F_{n, p}^{(k)}(\beta ; y)}{n+1},
$$

which means the asserted result (37).

Taking $p(n)=\frac{1}{\Gamma(n)}$ in (11) gives

$$
u_{k}\left(\log (1+z) \mid \frac{1}{\Gamma}\right)=\sum_{m=1}^{\infty} \frac{(\log (1+z))^{m}}{m^{k}(m-1) !},
$$

by which we get

$$
\begin{aligned}
\sum_{n=0}^{\infty} F_{n, \frac{1}{\Gamma}}^{(k)}(x ; y) \frac{z^{n}}{n !} & =\frac{1}{z\left(1-y\left(e^{z}-1\right)\right)} e^{x z} u_{k}\left(\log (1+z) \mid \frac{1}{\Gamma}\right) \\
& =\frac{e^{x z}}{z\left(1-y\left(e^{z}-1\right)\right)} \sum_{m=1}^{\infty} \frac{(\log (1+z))^{m}}{m^{k}(m-1) !} .
\end{aligned}
$$

Especially, for $k=1$ in (38), we obtain

$$
\sum_{n=0}^{\infty} F_{n, \frac{1}{\Gamma}}^{(1)}(x ; y) \frac{z^{n}}{n !}=\frac{e^{x z}}{z\left(1-y\left(e^{z}-1\right)\right)} \sum_{m=1}^{\infty} \frac{(\log (1+z))^{m}}{m !}=\sum_{n=0}^{\infty} F_{n}(x ; y) \frac{z^{n}}{n !},
$$

which gives the following equality

$$
F_{n, \frac{1}{\Gamma}}^{(1)}(x ; y)=F_{n}(x ; y) .
$$

Theorem 14. The following correlation

$$
F_{n, p}^{(k)}(x ; y)=\sum_{l=0}^{n} \sum_{m=0}^{l}\left(\begin{array}{l}
n \\
l
\end{array}\right) \frac{m ! p(m+1)}{(m+1)^{k-1}} \frac{F_{n-l}(x ; y)}{l+1} S_{1, \lambda}(l+1, m+1)
$$

holds for $n \geq 0$ and $k \in \mathbb{Z}$. Moreover, for $p(n)=\frac{1}{\Gamma(n)}$,

$$
F_{n, \frac{1}{\Gamma}}^{(k)}(x ; y)=\sum_{l=0}^{n} \sum_{m=0}^{l}\left(\begin{array}{l}
n \\
l
\end{array}\right) \frac{S_{1}(l+1, m+1)}{l+1} \frac{F_{n-l}(x ; y)}{(m+1)^{k-1}} .
$$

Proof. From (33), we have 


$$
\begin{aligned}
\sum_{n=0}^{\infty} F_{n, p}^{(k)}(x ; y) \frac{z^{n}}{n !} & =\frac{e^{x z}}{z\left(1-y\left(e^{z}-1\right)\right)} \sum_{m=1}^{\infty} \frac{(\log (1+z))^{m}}{m^{k}} p(m) \\
& =\frac{e^{x z}}{z\left(1-y\left(e^{z}-1\right)\right)} \sum_{m=0}^{\infty} \frac{(\log (1+z))^{m+1}}{(m+1)^{k}} p(m+1) \\
& =\frac{e^{x z}}{z\left(1-y\left(e^{z}-1\right)\right)} \sum_{m=0}^{\infty} \frac{m ! p(m+1)}{(m+1)^{k-1}} \sum_{l=m+1}^{\infty} S_{1}(l, m+1) \frac{z^{l}}{l !} \\
& =\sum_{n=0}^{\infty} F_{n}(x ; y) \frac{z^{n}}{n !} \sum_{m=0}^{\infty} \frac{p(m+1)(m+1) !}{(m+1)^{k}} \sum_{l=m}^{\infty} \frac{S_{1}(l+1, m+1)}{l+1} \frac{z^{l}}{l !} \\
& =\sum_{n=0}^{\infty}\left(\sum_{l=0}^{n} \sum_{m=0}^{l}\left(\begin{array}{l}
n \\
l
\end{array}\right) \frac{m ! p(m+1)}{(m+1)^{k-1}} \frac{F_{n-l}(x ; y)}{l+1} S_{1}(l+1, m+1)\right) \frac{z^{n}}{n !},
\end{aligned}
$$

which is the desired result (40).

Theorem 15. For $n \geq 0$ and $k \in \mathbb{Z}$, we have

$$
F_{n, p}^{(k)}(x ; y)=\sum_{l=0}^{n} \sum_{m=0}^{l}\left(\begin{array}{l}
n \\
l
\end{array}\right) F_{n-l, p}^{(k)}(y) S_{2}(l, m)(x)_{m} .
$$

Proof. By (33), we attain

$$
\begin{aligned}
\sum_{n=0}^{\infty} F_{n, p}^{(k)}(x ; y) \frac{z^{n}}{n !} & =\left(e^{z}-1+1\right)^{x} \frac{u_{k}(\log (1+z) \mid p)}{z\left(1-y\left(e^{z}-1\right)\right)} \\
& =\left(\sum_{m=0}^{\infty}(x)_{m} \frac{\left(e^{z}-1\right)^{m}}{m !}\right) \frac{u_{k}(\log (1+z) \mid p)}{z\left(1-y\left(e^{z}-1\right)\right)} \\
& =\left(\sum_{l=0}^{\infty} \sum_{m=0}^{l}(x)_{m} S_{2}(l, m) \frac{z^{l}}{l !}\right)\left(\sum_{n=0}^{\infty} F_{n, p}^{(k)}(y) \frac{z^{n}}{n !}\right) \\
& =\sum_{n=0}^{\infty}\left(\sum_{l=0}^{n} \sum_{m=0}^{l}\left(\begin{array}{l}
n \\
l
\end{array}\right) F_{n-l, p}^{(k)}(y) S_{2}(l, m)(x)_{m}\right) \frac{z^{n}}{n !},
\end{aligned}
$$

which provides the claimed result (42).

Lastly, we state the following theorem.

Theorem 16. Let $k \in \mathbb{Z}$ and $n \geq 0$. We have

$$
F_{n, p}^{(k)}(y)=\sum_{l=0}^{n} \sum_{r=0}^{n-l} \sum_{m=0}^{l}\left(\begin{array}{c}
n \\
l
\end{array}\right)\left(\begin{array}{c}
n-l \\
r
\end{array}\right) \frac{D_{r} F_{n-r-l}(y) S_{1}(l, m)}{(m+1)^{k}} p(m+1) m !,
$$

where $D_{r}$ is $r$-th Daehee number given by (cf. [18])

$$
\frac{\log (1+z)}{z}=\sum_{r=0}^{\infty} D_{r} \frac{z^{r}}{r !}
$$

Proof. From (14), (17), and (34), we have 


$$
\begin{gathered}
\sum_{n=0}^{\infty} F_{n, p}^{(k)}(y) \frac{z^{n}}{n !}=\frac{\sum_{m=1}^{\infty} \frac{p(m)}{m^{k}}(\log (1+z))^{m}}{z\left(1-y\left(e^{z}-1\right)\right)} \\
=\frac{\log (1+z)}{z} \frac{1}{1-y\left(e^{z}-1\right)} \sum_{m=0}^{\infty} \frac{p(m+1) m !}{(m+1)^{k}} \sum_{l=m}^{\infty} S_{1}(l, m) \frac{z^{l}}{l !} \\
=\sum_{r=0}^{\infty} D_{r} \frac{z^{r}}{r !} \sum_{n=0}^{\infty} F_{n}(y) \frac{z^{n}}{n !} \sum_{l=0}^{\infty}\left(\sum_{m=0}^{l} \frac{p(m+1) m !}{(m+1)^{k}} S_{1}(l, m) 2^{l}\right) \frac{z^{l}}{l !} \\
=\sum_{n=0}^{\infty} \sum_{r=0}^{n} \sum_{l=0}^{r}\left(\begin{array}{c}
n \\
r
\end{array}\right)\left(\begin{array}{l}
r \\
l
\end{array}\right) D_{r} F_{n-r-l}(y) \sum_{m=0}^{l} \frac{p(m+1) m !}{(m+1)^{k}} S_{1}(l, m) \frac{z^{n}}{n !} .
\end{gathered}
$$

Therefore, we obtain the claimed correlation (43).

\section{Conclusions}

Inspired and motivated by the definition of the type 2 poly-Bernoulli given by KimKim [12], in the present paper, we have introduced a new extension of the two-variable Fubini polynomials using the polyexponential function, which we call two-variable type 2 poly-Fubini polynomials. Then, we have acquired some useful relations including the Stirling numbers of the first and the second kinds, the Bernoulli polynomials of higher-order, and the usual Fubini polynomials. Also, we have developed some summation formulas and an integral representation for type 2 poly-Fubini polynomials. Moreover, we have considered two-variable unipoly-Fubini polynomials via unipoly function and have investigated diverse properties including derivative and integral properties. Furthermore, we have provided some relationships covering the two-variable unipoly-Fubini polynomials, the Stirling numbers of the first and the second kinds, and the Daehee polynomials.

Author Contributions: Writing—original draft, G.M., W.A.K. and U.D.; Writing—review \& editing, G.M., W.A.K. and U.D. All authors have read and agreed to the published version of the manuscript.

Funding: This research received no external funding.

Institutional Review Board Statement: Not applicable.

Informed Consent Statement: Not applicable.

Data Availability Statement: Not applicable.

Conflicts of Interest: The authors declare no conflict of interest.

\section{References}

1. Duran, U.; Acikgoz, M. On degenerate truncated special polynomials. Mathematics 2020, 8, 144. [CrossRef]

2. Guohui, C.; Li, C. Some identities involving the Fubini polynomials and Euler polynomials. Mathematics 2018, 6, 300. [CrossRef]

3. Kargin, L. Exponential polynomials and its applications to the related polynomials and numbers. arXiv 2016, arXiv:1503.05444v2.

4. Kilar, N.; Simsek, Y. A new family of Fubini type numbers and polynomials associated with Apostol-Bernoulli numbers and polynomials. J. Korean Math. Soc. 2017, 54, 1605-1621.

5. Kwon, J.; Jang, L.C. A note on the type 2 poly-Apostol-Bernoulli polynomials. Proc. Jangjeon Math. Soc. 2020, 30, $253-262$.

6. Ma, Y.; Kim, D.S.; Lee, H.; Kim, T. Poly-Dedekind sums associated with poly-Bernoulli functions. J. Inequal. Appl. 2020, $2020,248$. [CrossRef]

7. Duran, U.; Acikgoz, M. Truncated Fubini polynomials. Mathematics 2019, 7, 431. [CrossRef]

8. Kim, D.S.; Kim, T.; Kwon, H.-I.; Park, J.-W. Two-variable higher-order Fubini polynomials. J. Korean Math. Soc. 2018, 55, 975-986. [CrossRef]

9. Sharma, S.K.; Khan, W.A.; Ryoo, C.S. A parametric kind of the degenerate Fubini numbers and polynomials. Mathematics 2020, 8 , 405. [CrossRef]

10. Su, D.D.; He, Y. Some identities for the two-variable Fubini polynomials. Mathematics 2019, 7, 115. [CrossRef]

11. Duran, U.; Acikgoz, M.; Araci, S. Construction of the type 2 poly-Frobenius-Genocchi polynomials with their certain applications. Adv. Differ. Equ. 2020, 2020, 432. [CrossRef]

12. Kim, D.S.; Kim, T. A note on polyexponential and unipoly functions. Russ. J. Math. Phys. 2019, 26, 40-49. [CrossRef]

13. Kim, T.; Kim, D.S.; Lee, H.; Jang, L.-C. Identities on poly-Dedekind sums. Adv. Differ. Equ. 2020, 2020, 563. [CrossRef] 
14. Eastham, M.S.P. On Polylogarithms. Proc. Glasgow Math. Assoc. 1964, 6, 169-171. [CrossRef]

15. Lewin, L. Polylogarithms and Associated Functions; With a Foreword by Vander Poorten, A.J.; North-Holland Publishing Co.: Amsterdam, NY, USA, 1981.

16. Dolgy, D.V.; Jang, L.C. A note on the polyexponential Genocchi polynomials and numbers. Symmetry 2020, 12, 1007. [CrossRef]

17. Lee, D.S.; Kim, H.K.; Jang, L.-C. Type 2 degenerate poly-Euler polynomials. Symmetry 2020, 12, 1011. [CrossRef]

18. Khan, W.A.; Nisar, K.S.; Duran, U.; Acikgoz, M.; Araci, S. Multifarious implicit summation formulae of Hermite-based poly-Daehee polynomials. Appl. Math. Inf. Sci. 2018, 12, 305-310. [CrossRef] 